\title{
Schedule-dependent recovery of barpressing performance in frontal rats*
}

\author{
S. D. GLICK and R. G. MARSANICO \\ Department of Pharmacology, Mount Sinai School of Medicine \\ 100th Street and Fifth Avenue, New York, New York 10029 \\ and \\ Herbert M. Singer Laboratory of Neurosciences and Addictive Diseases, Beth Israel Medical Center \\ 307 Second Avenue, New York, New York 10003
}

\begin{abstract}
Rats with bilateral frontal cortical ablations showed early postoperative rate decrements on either a FI 15 or FR 30 schedule of barpressing for water. Although the performance of all frontal rats recovered to some extent during a month of postoperative testing, complete recovery occurred only in rats tested on the FI 15 schedule. Observations of barpressing behavior indicated that FR 30 barpressing required a degree of bimanual motor coordination not required in normal FI 15 barpressing. A persistent disruption of such coordination by frontal cortical lesions appeared to account for the differential recovery of barpressing rates on the two schedules.
\end{abstract}

Depressed rates of operant barpressing performance have been reported, though only occasionally (Glick, 1969; Campbell \& Lynch, 1969; Schmaltz \& Isaacson, 1972), following bilateral ablations of frontal cortex in rats. Initial observations of CRF (continuous reinforcement) responding made in this laboratory indicated that rapid recovery of rates to a normal baseline occurred as a function of time and/or continual testing after surgery. The present study was an attempt to determine if the schedule of reinforcement maintaining barpressing affects the magnitude of the postoperative deficit or the degree of eventual recovery.

\section{METHOD}

\section{Subjects}

The Ss were 12 naive female Sprague-Dawley rats approximately 3 months old at the beginning of the experiment.

\section{Surgery and Histology}

All surgery was conducted under methohexital anesthesia. A scalp incision was made along the midline of the head, and subcutaneous tissue was deflected. With the use of a dental drill, bilateral burr holes, each $5 \mathrm{~mm}$ in diam, were made in the skull, $0.5 \mathrm{~mm}$ from the midline and $0.5 \mathrm{~mm}$ in front of the coronal suture. This procedure alone constituted the sham operation. Frontal cortex was removed by suction through a 20 -ga needle under a dissecting microscope. Both sham and frontal operations were completed by closing the wound with $7-\mathrm{mm}$ wound clips.

Following their use in the experiment, all rats were killed and perfused with $10 \%$ Formalin. Their brains were removed and immersed in Formalin for at least a week before sections ( 40 microns stained with Luxol blue and cresyl violet) were made and histological examination was conducted. Lashley diagrams, whole brain photographs, and microphotographs of frontal ablations extremely similar to those used in this study have been published previously (Glick, 1969, 1971; Glick \& Greenstein, 1973). Briefly, the major area of destruction was confined to frontal cortex. Only rarely was subcortical white matter included in the lesion. The major area of cortex

*This research was supported by NIMH Grant MH21156 and NIMH Research Scientist Development Award (Type 2) DA70082 to S. D. Glick. destroyed extended rostrally to the tip of the frontal poles and caudally to the anterior limb of the corpus callosum.

\section{Apparatus and Procedure}

All testing was conducted in four Lehigh Valley operant test cages (No. 143-241), each enclosed in a sound-attenuated cubicle (No. 132-02). Responses on either of two levers in each test cage were rewarded with water $(0.01 \mathrm{ml})$ and recorded on Sodeco counters.

Rats were deprived of water for 2 days prior to the beginning of preoperative testing. Each rat was placed in a test cage for one 30-min daily session. Additional water was provided in each rat's home cage for $15 \mathrm{~min}$ after testing. Testing was conducted 5 days a week (Monday-Friday). After testing on Friday, water was provided ad lib until Saturday morning; rats were then deprived until testing on Monday. Initially, each rat was shaped by delivering a noncontingent water reward every minute during testing; every barpress was also rewarded with water. During this shaping period, each reward presentation was also accompanied by the onset of light situated above the water dipper. When a rat made a total of 100 responses (both levers combined) during a test session, the light as well as the noncontingent water reward were eliminated from the schedule. This procedure took approximately 7 test sessions for each rat. Rats were then maintained on a schedule of continuous reinforcement for 7 more sessions. For six rats, a fixed-interval 5-sec (FI 5) schedule was then begun; a response on either lever was rewarded every 5 sec. For the other six rats, a fixed-ratio 4 (FR 4) schedule was begun; every fourth response was rewarded. The FI schedule was subsequently changed to FI 10 and finally to FI 15 . The FR schedule was subsequently changed to FR 8,16 , and finally to FR 30. The progression for each schedule entailed 12 test sessions. After another 10 test sessions, the rates of all rats had stabilized, varying no more than $\pm 10 \%$ from the first session within a 4-session period (there were absolutely no consistent or significant differences between Mondays and Fridays). For the next two sessions, all rats were tested with the door of the sound-attenuated cubicle open. This was done to allow observation of how each rat barpressed. After another 15 test sessions of asymptotic performance, surgery was performed on all rats (four frontal FR, four frontal FI, two sham FR, and two sham FI).

Daily testing was continued, as before surgery, on the day following surgery and for 5 weeks thereafter. During the sixth postoperative week, all rats were again tested and observed with the door of the sound-attenuated cubicle open. 
Table 1

Individual Pre- and Postoperative FI 15 Barpressing Rates (Responses Per $30 \mathrm{Min}$ )

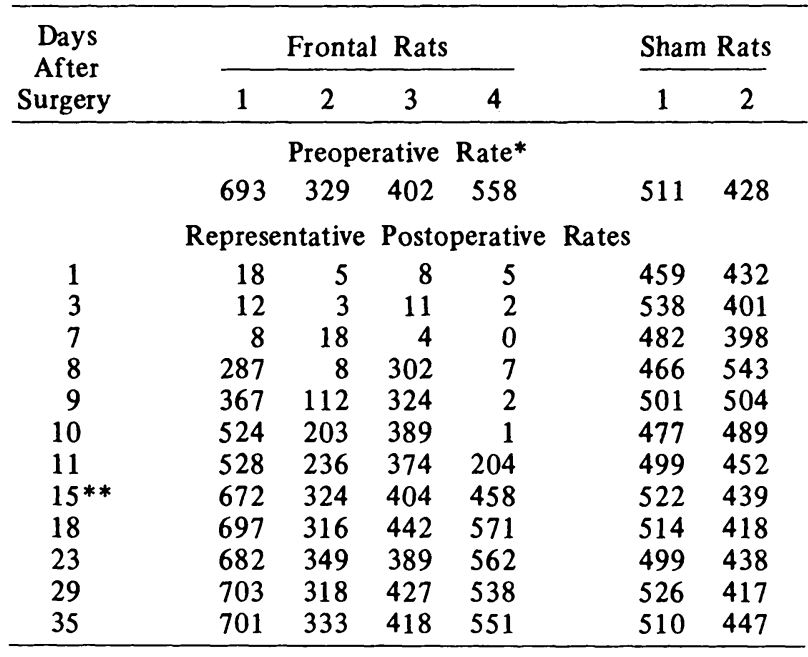

* Mean rates during week preceding surgery.

**Frontal effect significant prior to but not on or after Day 15 ( $p>.05$, paired preoperative-postoperative $t$ tests for whole group).

Table 2

Individual Pre- and Postoperative FR 30 Barpressing Rates (Responses Per $30 \mathrm{Min}$ )

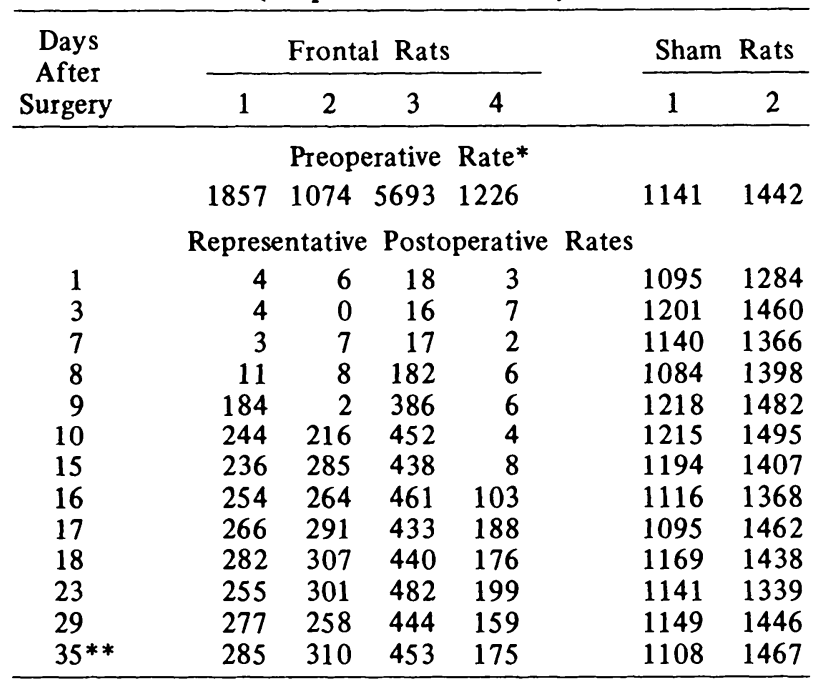

*Mean rates during week preceding surgery.

**Frontal effect significant on every day up to and including Day 35 ( $p<.001$, paired preoperative-postoperative $t$ tests for whole group.).

\section{RESULTS AND DISCUSSION}

Tatles 1 and 2 show the pre- and postoperative rate data for each individual rat. Following surgery, the rates of all frontal rats were depressed. To some extent, all rates recovered in the month following surgery. However, whereas the FI frontal rats all recovered fully, no FR frontal rats ever recovered completely (i.e., postoperative rates never became as high as the mean preoperative rate). Two observations are important: (1) In all cases, recovery appeared to be dependent on time per se after surgery rather than on additional postoperative training; the most common pattern of recovery was an almost total cessation of barpressing for several days after surgery followed by an abrupt return to barpressing at a near asymptotic level. (2) There were very obvious differences observed in the ways in which normal rats used their paws to respond on the two different schedules. Normal FR rats used both paws in alternating fashion to make all responses, i.e., high FR rates were generated by using a rapid alternating left-right-left-right, etc., paw sequence to press the lever. In contrast, normal FI rats used either one paw alone or both paws simultaneously (both paws placed on lever together rather than in alternating sequence) to make all responses, i.e., lower FI rates were generated by discrete movements requiring less bimanual coordination. Frontal lesions were found to have very different effects on the kinds of paw usage during FI and FR responding. When observed during the sixth postoperative week, all frontal rats used either one paw alone or both paws simultaneously to make all responses. Although attempts were noted, there was a marked absence and an apparent inability to perform the paw alternation formerly used in preoperative FR responding.

The effect of frontal lesions on paw usage appears to account for the differential FI and FR recovery results. The early postoperative effects may be due to a combination of motor and motivational deficits recovering as a function of time after surgery (Glick \& Greenstein, 1973). Persisting, however, is a residual inability to coordinate fine bimanual movements. Thus, the extent of recovery in a particular task will depend upon the importance of such a residual deficit in performance of that task. It is also evident that similar kinds of behavior recorded automatically may be accomplished in very different ways (Peterson, Ackil, Frommer, \& Hearst, 1972). It is anticipated that further analyses of this sort will be helpful in understanding other cases of differential recovery following similar lesions (e.g., Lynch, Ballantine, \& Campbell, 1971).

\section{REFERENCES}

Campbell, B. A., \& Lynch, G. S. Cortical modulation of spontaneous activity during hunger and thirst. Journal of Comparative \& Physiological Psychology, 1969, 67, 15-22.

Glick, S. D. Discrimination learning and reversal in frontal rats as a function of cue. Physiology \& Behavior, 1969, 4, 389-392.

Glick, S. D. Modulation of food and water intake by frontal cortex in the rat. Communications in Behavioral Biology, $1971,5,365-370$.

Glick, S. D., \& Greenstein, S. Recovery of weight regulation following ablation of frontal cortex in rats. Physiology \& Behavior, 1973, 10, 491-496.

Lynch, G., Ballantine, P., \& Campbell, B. A. Differential rates of recovery following frontal cortical lesions in rats. Physiology \& Behavior, $1971,7,737-741$.

Peterson, G. B. Ackil, J. E. Frommer, G. P. \& Hearst, J. E. Conditioned approach and contact behavior toward signals for food or brain-stimulation reinforcement. Science, 1972, 177, 1009-1011.

Schmaltz, L. W., \& Isaacson, R. L. Effect of caudate and frontal lesions on acquisition and extinction of an operant response. Physiology \& Behavior, 1972, $9,155-159$.

(Received for publication October 25, 1973; revision received December $6,1973$. 